\title{
Die Petruserzählungen im Markusevangelium.
}

Von M. Brückner in Karlsruhe.

Es ist eine in der Evangelienkritik fast zum Dogma gewordene Annahme, $\mathrm{da} b$ in unserm Markusevangelium Petruserzählungen enthalten seien, die seinem Inhalte mehr oder weniger die Bürgschaft urapostolischer Tradition gewähren.

Diese Annahme beruht auf der bekannten Papiasnotiz bei Eusebius (K. G. III, 39, 15), nach der Markus Worte und Taten Jesu auf Grund von Erinnerungen aus seiner früheren Gefolgschaft des Apostels Petrus aufgezeichnet habe. Papias selbst beruft sich dabei auf das mündliche Zeugnis des "Presbyters" als seines Gewährsmannes. Diese Tradition gibt Irenäus (Euseb V. 8, 2) mit der ausdrücklichen Bemerkung weiter, $\mathrm{da} B$ Markus seine Aufzeichnungen erst nach dem Heimgange des Petrus und des Paulus gemacht habe, während Clemens Alexandrinus (Euseb VI, 14, 5) ihn sein "Evangelium" noch zu Lebzeiten des Petrus, auf Bitten einiger Hörer des Apostels, niederschreiben läßt. Bei letzterem scheint doch deutlich eine Fortbildung der Tradition zu Gunsten einer noch erhöhten apostolischen Autorität des Markus vorzuliegen. Joh. Weiß (Das älteste Evangelium 1902. S. 350) findet allerdings in beiden Mitteilungen „etwas Richtiges“. Clemens spreche von den Aufzeichnúngen, die sich Markus "naturgemäß" schon damals gemacht habe, als er den greisen Petrus noch hören konnte, während Irenäus „die definitive Veröffentlichung des Evangeliums" meine. In Wahrheit meinen aber beide unser Markusevangeliụm; und da auch schon die Papiasnotiz für die Aufzeichnungen des Markus den Tod des Petrus deutlich voraussetzt, so dürfte der Harmonisierungsversuch von Joh. Weiß schwerlich Beifall finden. Vielmehr muß diese Fortbildung der Tradition gegen diese selbst bedenklich machen. Die Begründung durch apostolische Tradition mußte ja das Markusevangelium früher oder später bekommen, wenn es überhaupt kirchliche Geltung erhalten sollte. Die Tatsache solcher Begründung ist daher an sich nicht im geringsten schon die 
Bürgschaft ihrer geschichtlichen Richtigkeit. Sie ist es zumal dann nicht, wenn sie, wie in unserm Falle, erst auf dritter Hand beruht und von einem Papias herrührt, der bekanntlich auch sonst kein einwandsfreier Zeuge ist und von Eusebius als ein Mann $c థ \delta \delta \rho \alpha$ c $\mu$ เкрòc tòv voûv bezeichnet wird. E. Schwartz spricht daher auch dieser Tradition jeden selbständigen Wert ab (Über den Tod der Söhne Zebedäi. Abhandlg. d. Götting. Ges. d. Wissensch. N. F. VII, 5. S. 22).

In der Tat glaubt es ja auch niemand dem Papias mehr, dab unser Markusevangelium selbst die Aufzeichnung jener Petruserinnerungen des Markus sei. Sondern man führt, wie auch Joh. Weiß (S. 349) ausdrücklich bekennt, gegen den Wortlaut und wahrscheinlich auch gegen die Meinung des Papias nur einen Teil der im zweiten Evangelium enthaltenen Überlieferungen auf Petruserzählungen zurück. Damit verliert aber die Papiasnotiz auch jeden sachlichen Wert für die Begründung dieser Hypothese. Denn sie enthält ja nicht die geringste Andeutung über Umfang und Inhalt der Petruserzählungen. Sie hat nur noch den Wert einer recht fraglich begründeten und inhaltlich unbestimmten Behauptung, die erst und ganz allein aus dem Markusevangelium selbst bewiesen werden müßte. Dieser Tatbestand wird auch prinzipiell anerkannt; faktisch aber rechnet man mit der Tradition des Papias wie mit einer feststehenden Tatsache. Demgegenüber ist es nicht überflüssig $z u$ betonen, daß wir zur Feststellung von etwa vorhandenen Petruserzählungen lediglich auf ihre Selbstbezeugung im Markusevangelium angewiesen sind.

Es soll nun im Folgenden gezeigt werden, daß den von der Kritik als Petruserzählungen in Anspruch genommenen Stücken die genügende wissenschaftliche Begründung fehlt. Ihr Inhalt und Charakter läßt vielmehr eine derartige Annahme als höchst unwahrscheinlich gelten. Dagegen gibt es im Markusevangelium „Petruserzählungen“, die sich als solche selbst bezeugen. Es sind die Geschichten und Reden, bei denen nur die besonderen Vertrauten Jesu zugegen gewesen sein sollen: Die Auferweckung der Tochter des Jairus C. 5, 37 ff.; die Verklärung Jesu C. 9, 2 ff.; die Zukunftsrede C. 13,3 ff.; Gethsemane C. 14, 33 ff. In allen diesen Geschichten steht Petrus als Gewährsmann an erster Stelle. Wir werden daher nicht fehlgehen, wenn wir grade in ihnen den Ursprung der Tradition aller Petruserzählungen suchen. Es wird sich zeigen, daß wir es hier mit Traditionen geschichtsloser Herkunft $z u$ tun haben, die durch den Namen des Petrus zu geschichtlicher Glaubwürdigkeit erhoben werden sollten.

Zeitschr. L. d. neutest. Wiss. Jahrg. VIII. 1907. 
Dic Feststellung von etwa vorhandenen Petruserzählungen im Markusevangelium ist von vornhercin durch die Komposition desselben außerordentlich erschwert. Denn, wenn es sich auch schon einer oberflächlichen Betrachtung zeigt, dali dieses Evangelium kein Werk aus einem Guß, sondern dic Zusammenstellung und Bearbeitung eines mannigfachen und verschiedenen Schichten der Entwicklung angehörigen Traditionsstoffes ist, so ist doch diese Bearbeitung nach bestimmten Gesichtspunkten und so durchgreifend erfolgt, daß eine Quellenscheidung nach literarkritischer Methode bisher noch nicht gelungen ist. ${ }^{x}$ An sich ist es z. B. nicht unwahrscheinlich, daß schon dem ersten Zusammensteller des Stoffes schriftlich fixierte Traditionsstücke und Gruppen vorlagen; aber ein überzeugender Nachweis solcher ist scheinbar aussichtslos, zumal wenn man bedenkt, daß unser heutiger Markus vielleicht aus der Übersetzung eines aramäischen Originals mit allerhand Zutaten und Umarbeitungen entstanden ist.

Bei einem solchen Werke kann die Literarkritik nur gẹringe Helferdienste leisten. Die dem Tatbestande entsprechendste Methode ist vielmelir diejenige, die nach den sachlichen Gesichtspunkten fragt, die zur Aufnahme und Ausgestaltung des Stoffes geführt haben. Als glänzendes Beispiel dieser Methode ist Wredes "Messiasgeheimnis" zu nennen. Hier hat Wrede in der Tat einen die Tradition um- und neubildenden Gedanken aufgezeigt, der sich als ein brauchbares Mittel geschichtlicher Kritik erweist, mag ihn der Verfasser auch etwas zu einseitig betont haben. Aber diese Kritik erstreckt sich direkt auf die Geschichtlichkeit des Stoffes selbst, ohne die Frage nach dessen literarischer Herkunft $z u$ betonen. Einen anderen, nicht minder wichtigen Gesichtspunkt hat Wellhausen in dem Nachweise eines doppelten Messiasbildes bei Markus geltend gemacht. Hierdurch wird, auch wieder ohne Rücksicht auf die literarische Frage, zunächst rein sachlich der ganze "Jüngerabschnitt" (C. 8-10) als eine jüngere Schicht der Tradition gekennzeichnet. Wir kommen unten noch darauf zurück.

Es erweckt nach dem Gesagten von vornherein kein günstiges Vorurteil, da $\$$ bei der Ausscheidung der vermeintlichen Petrusstücke vor allem der literarkritische Gesichtspunkt zur Geltung kommt, v. Soden beruft sich in seiner Schrift über die wichtigsten Fragen im Leben Jesu

I Auch der neueste Versuch von E. Wendling (Ur-Marcus. 1905. Tübingen, J. C. B. Mohr), der (S. 20) einen Historiker MI, einen Poëten $\mathrm{NI}_{2}$ und einen Dogmatiker Ev. in unserm Evangelinm unterscheiden will, muß schon wegen dieses Schematismus als gescheitert gelten. 
(Berlin, Duncker 1904. S. 22) überhaupt nur darauf, daß sich nicht alle Erzählungen „in die Struktur des Evangeliums“ fügen, und daß „die große Verschiedenheit der Erzählungsweise zwei Erzähler" verrate. Die Petruserzählungen „heben sich so scharf $a b$, daß ihre Auslösung keine irgend nennenswerte Schwierigkeiten macht". v. Soden rechnet dazu folgende Stücke:

I. Johannes der Täufer und die Taufe Jesu.

C. I, 4-II.

2. Ein Sabbat in Kapernaum.

C. I, 2I-39.

3. An was die Juden sich stießen.

C. 2, I-12: Daß Jesus Sündenvergebung ausspricht.

C. 2, I3-I7: $\mathrm{Dab}$ er mit Sündern verkehrte.

C. 2, 18-22: $\mathrm{DaB}$ er nicht fastete.

C. 2, 23-3, 6: $\mathrm{Dab}$ er die Sabbatvorschriften zurückstellte.

4. Wie sie ihn zu fangen versuchten.

C. $12,13-44$.

5. Wie Jesus überall Verständnislosigkeit begegnete.

6. Gleichnislehre vom Gottesreich.

(C. 4, I-9. 2I-32.)

7. Wer kommt ins Gottesreich?

(C. 10, 13-45.)

8. Die Entwicklung des Jüngerkreises.

C. 1, 16-20: Die Wahl der Vier.

C. 3, 13-19: Die Wahl der Zwölf.

C. 6,7-16: Die Instruktion der Zwölf.

C. 8, 27-9, I: Das Messiasbekenntnis der Zwölf.

C. 9, 33-40: Gegen die Ausnahmestellung der Zwölf.

9. Blicke in die Zukunft.

C. $13,1-6.28-37$.

$\mathrm{Da}$ sich alle diese Stücke leicht aus ihrem Zusammenhange herausnehmen lassen, ist richtig, besagt aber gar nichts. Denn man kann auch alle andern Stücke bis zur Leidensgeschichte so herausnehmen, da sie fast alle olne sachlichen und zeitlichen Zusammenhang aneinander gereiht sind. Man kann auch die meisten Stuicke beliebig umstellen, ohne die vermeintliche "Struktur“ des Evangéliums zu zerstören. Denn die Struktur, die v. Soden in dem ersten Hauptteile findet, ist in der Tat nur eine vermeintliche. v. Soden unterscheidet $\mathrm{I}$. die erste Zeit C. $1,16-3,6$; 2. die Steigerung der Bewegung C. 3, 7-5, 43; 3. den 
Massenerfolg C. $6,1-8,26 ; 4$. dic Beschränkung auf die Zwölf C. 8, $27-9,50$. Abcr diese Unterschcidung trifft nicht $z u$, da Jesus von Anfang an Massencrfolg liat C. 1, 28. 45. 2, 2. 13 und diesen Erfolg auch bis zuletzt behält C. 8, 34. 14. 10, 1. Vor allem ist von einer Steigerung der Bewegung nirgends die Rede. Das Gleichnis vom Säemann, das nach v. Sodens "Struktur" unter diesem Abschnitt steht, redet doch mehr von Enttäuschung als von Erfolg. Trotzdem palst es sachlich an seine Stelle ebenso gut oder schlecht, wie es z. B. hinter C. 1 oder 10 stehen könnte. In der "Struktur" des Evangeliums liegt gar keine Veranlassung, es aus dem gegenwärtigen Zusammenhange zu entfernen. Und das gilt nicht nur für das Säemannsgleichnis: die eigenartige Komposition des Evangeliums verbietet es fast überall, aus seiner "Struktur" Schlüsse über die ursprüngliche Zugehörigkeit einzelner Stücke zu ziehen. Man kann damit fast alles und folglich nichts beweisen.

Auch die Erzählungsarten sind im Markusevangelium gewiß verschieden, da es ein Sammelwerk von verschiedenartigen Traditionsstoffen ist. $\mathrm{Ob}$ sich aber eine Reihe von Stuicken so gleichmäßig von den andern unterscheidet, daß wir sie als Petruserzählungen in Anspruch nehmen dürfen, ist doch eine ganz andere Frage, deren Bejahung ganz bestimmte gemeinsame Merkmale in diesen Stücken voraussetzt. Was aber v. Soden dafür ausgibt (S. 37f.), ist völlig unzureichend. "Plastisch-konkret in jedem Zuge," "leuchtend frisches Lokalkolorit," "unerfindlich originell" - das alles sind doch ganz allgemeine $Z$ üge, die für Alter und Herkunft gar nichts beweisen und auf manche andere Stücke, wie z. B. auf die Speisungsgeschichte C. 6, 34ff. oder auf die vom Gerasener Besessenen C. 5, Iff. viel besser passen als auf viele der vermeintlichen Petruserzählungen. Die Heilung des letzteren rechnet v. Soden allerdings zu den drei "Großwundern" C. 4, 35-5, 43. Es ist aber doch wirklich nicht einzusehen, warum die Heilung eines Besessenen draußen ein so viel größeres Wunder sein soll, als in der Synagoge C. I, 2Iff.

Aber auch die wenigen konkreten Merkmale, die v. Soden anführt, sind nicht stichhaltig. Daß sich in den genannten Stücken "nie ein Anklang an alttestamentliche Geschichten" fände, ist einfach nicht richtig. Ich erinnere nur an die Berufung der beiden Brüderpaare C. I, I6ff., die auch nach Holtzmann (H.C.3 S. 49) gradezu eine Neubildung der Berufungsgeschichte des Elisa I. Kön I9, Igff. ist. Wenn v. Soden weiter darauf hinweist, daß in seinen Petrusstiicken Wunder „nur gelegentlich und ganz beiläufig" erscheinen, so liegt das nur an seiner vorsichtigen 
Ausivahl. Und die Wunder an dem Gelähmten C. 2, I ff. und dem Menschen mit der verdorrten Hand C. 3, Iff. sind durchaus nicht ganz beiläufig; so gut wie diese könnten dann auch noch andere zu den Petrusstücken gehören. Die Erzählung von dem epileptischen Knaben C. 9, I4ff. z. B. müßte nach den v. Sodenschen Merkmalen unbedingt dazu gerechnet werden; es ist willkürlich, sie auszulassen. Endlich trägt auch die Gestalt Jesu in diesen Stücken durchaus nicht, in jeder Beziehung menschliche Umrisse'c. Denn er verrichtet neben den doch auch nicht ganz zufälligen Heilungen in C. I, 2 I ff. Wunder mit so absoluter Gewißheit des Erfolges, daß darin das Maß des 'mènschlich Möglichen durchaus überschritten erscheint C. 2, I ff. 3, I ff. Auch hat er nicht nur von Anfang an das volle Messiasbewußtsein, sondern wei $\emptyset$ auch über den Ausgang seines Lebens von vornherein Bescheid C. 2, 19. 8, 31. Io, $38 \mathrm{ff}$.

Die Merkmale, die v. Soden zur Begründung seiner Petrusstücke anführt, reichen daher entfernt nicht aus, deren Aussonderung aus dem Zusammenhange des Evangeliums zu rechtfertigen. Sie schließen diese Stücke weder genügend von den andern $a b$, noch in sich zusammen. Vor allem aber fehlt diesen Stücken grade das, was sie erst als Petruserinnerungen kennzeichnen würde: das Merkmal persönlicher Beziehungen des Jüngers zu seinem Meister. Man hat, wie Wellhausen treffend bemerkt (Einleitung zu den drei ersten Evangelien S. 51), nicht den Eindruck, daß ein Versuch derer, die mit ihm gegessen und getrunken hatten, vorläge, anderen eine Anschauung von seiner Person zu geben.

Diesen Anspruch macht nun allerdings Joh. Weiß für seine Auswahl ausdrücklich geltend, wenn er als „die spezifischen Merkmale. der Petrusstücke“ (a. a. O. S. 364) angibt: „Die Gruppen, den frischen Erzählungscharakter, die persönlich gefärbten Erinnerungen." Aber wie fühlt man sich enttäuscht, grade bezüglich des letzteren Merkmales unter dem Titel "Charakter der Petruserzählungen" fclgendes Geständnis bei ihm (S. 360 ) zu lesen: „Da wir nun nur eine Auswahl besonders bedeutsamer Vorgänge won Markus erzählt finden, so fehlt diesen Berichten das, was man häufig von -ihnen erwartet oder gar in ihnen gefunden hat, das Tagebuchartige, das biographische Detail. Wir werden nicht in den alltäglichen Verkehr der Jünger mit dem Meister eingeführt, wir beobachten nichts weniger als den stillen, allmählichen, umbildenden Einfluß des Herrn; es sind lauter höchst aktuelle, ungewöhnliche Ereignisse, die uns mitgeteilt werden, lauter Höhepunkte dieses Zusammenlebens, in denen die Macht und Größe Jesu überwältigend hervortritt, oder in denen den 
Jüngern besondere Orfenbarungen zuteil werden; Wundertaten und messianische Kundgebungen, Konfliktc mit den Gegnern und erregte Momente aus dem Jüngerleben; dramatische Volksszenen und kritische Wendepunktc im öffentlichen Wirken Jesu - das ist der Inhalt dieser Stücke." - Ich mcine doch, das ist so ziemlich das Gegenteil von "persönlich gefärbten Etinnerungen". Und in der Tat: überblickt man die von Joh. Weiß getroffene Ausivahl, so findet man, daß sie in bezug auf persönliche Färbung nichts vor der v. Sodenschen voraus hat. Joh. Weif gibt (S. 350 f.) folgende Zusammenstellung:

I. Jesu Auftreten in Galiläa und die Berufung der vier Fischer.

2. Der Sabbat in Kapernaum.

3. Der Gichtbrüchige.

4. Volksandrang und Lästerung des Geistes.

5. Die wahren Verwandten?

6. Seepredigt, Ưberfahrt, Stillung des Sturmes, Gerasa, Iairi Töchterlein.

7. Verwerfung in Nazareth.

8. 1. Speisung, Überfạhrt, Seewandeln Jesu, Landung in Gennezareth, Zeichenforderung -

9. Nordreise, Petrusbekenntnis, Verklärung, Heilung des Knaben, 2. Leidensverkündigung.

10. Rangstreit (Lohnfrage des Petrus?), Ehrgeiz der Zebedaiden.

II. Einzug in Jerusalem. Zinsgroschenfrage.

12. Tempelreinigung. Vollmachtsfrage.

13. Gespräch über den Davididen. Wort über den Tempel?

14. (Verrat des Judas?), Gethsemane, Verleugnung, Verhör vor Pilatus, Kreuzigung.

Diese Auswahl der Petruserinnerungen unterscheidet sich von der, die v. Soden getroffen hat, abgesehen von der Hereinziehung der Leidensgeschichte hauptsächlich durch die Hinzufügung der unter 6, 8 und 9 angeführten "Gruppen". Aber grade in diesen Gruppen ist außer in dem auch von v. Soden angezogenen Petrusbekenntnisse von persönlich ge- färbten Erinnerungen des Petrus nichts zu spüren. Wir fragen vielmehr mit Wellhausen: Soll etwa Petrus das Wandeln auf dem See oder das Ausfahren der bösen Geister in die Säue bezeugt haben, die Heilung des blutflüssigen Weibes durch die Kraft eines Kleides? Joh. Weiß traut dem Petrus allerdings das alles zu (S. 364), während er andrerseits die meisten Wunder rationalistisch wegdeutet. Im Grunde hat er aber für die Beschlagnahme dieser Erzählungen als Petrusstücke keine andere 
Begründung als die, daß sie schon vor ihrer Aufnahme ins Markusevangelium zu festen Gruppen vereinigt waren. Das letztere ist mir für die Gruppen $\delta$ und 9 allerdings wegen des parallelen Zusammenhanges der beiden Speisungsgeschichten auch wahrscheinlich. Aber ist damit irgend ein Beweis geliefert, daß sie von Petrus herrühren? Kann sich dieser feste Zusammenhang nicht ebensogut in volkstümlicher Überlieferung gebildet haben? Mag man auch, wie Joh. Weiß offen gesteht (S. 120ff.), mit dem ausdrücklichen Vorurteil an das Markusevangelium herantreten, Petruserzählungen darin zu finden, immer kommt es doch für den Beweis zuletzt darauf an, daß diese Erzällungen irgendwie eine besondere persönliche Färbung tragen. Das ist aber bei den genannten Gruppen nicht der Fall. Es wird aber fast allgemein von zwei Stücken behauptet, die wir deshalb noch einer besonderen Betrachtung unterziehen müssen. Es sind die Geschichten von dem ersten Auftreten Jesu in Kapernaum C. $\mathrm{I}, 16 \mathrm{ff}$. und dem Petrusbekenntnis C. 8, $27 \mathrm{ff}$.

$\mathrm{Zu}$ dem Abschnitte Mc I, I6ff. sagt $\mathrm{H}$. J. Holtzmann noch in seiner neuen Bearbeitung des Handkommentars S. II, daß hier „der Eröffnungstag der Wirksamkeit Jesu und der Rückschlag, welchen die erlebten Erfolge am andern Morgen nach sich ziehen, mit einer Anschaulichkeit geschildert seien, der sich fast nur die Berichte über die letzten Tage Jesu an die Seite stellen können".

Aber es ist die Anschaulichkeit der Dichtung, nicht der Geschichte. Sie schwindet, sobald man es ernstlich versucht, sich in die Situation zu versetzen, und man gewahrt deutlich die treibenden Kräfte und Anschauungen, die diese Szenen geschaffen haben.

Der Abschnitt will den Eröffnungstag der Wirksamkeit Jesu schildern. $\mathrm{Da}$ es ein Sabbat ist, so kann die Berufung der vier arbeitenden Schiffer ursprünglich nicht mit dazu gehört haben. Sie wird aber in V. $29 \mathrm{ff}$. doch wieder sachlich vorausgesetzt, weil Jesus mit jenen Vier aus der Synagoge in das Haus des Simon geht. Also ist die Nennung der Vier in V. 29 nicht geschichtlich, sondern bringt nur diese Geschichte mit der Berufung der Brüderpaare V. I $6 \mathrm{ff}$. schriftstellerisch in Zusammenhang: 'Wir fragen aber weiter: Von wo ḳommt Jesus in die Synagoge? Wo hat er die Nacht vom Freitag zum Sabbat zugebracht? Die Frage ist deshalb nicht gleichgültig, weil V. $29 \mathrm{ff}$. seinen Aufenthalt im Hause des Petrus ausschließt. Denn sonst hätte. er von der Krankheit der Schwiegermutter Simons ja schon wissen müssen. Da V. 29ff. andrerseits seine Bekanntschaft mit Petrus voraussetzt, so ist ein andrer Aufenthalt Jesu in Kapernaum nicht anzunehmen, und von außerhalb konnte 
er am Sabbat auch nicht gekommen sein. Also gibt die Chronologie der Erzählungen durchaus kein falibares, anschauliches Bild des Eröfnungstages. Die Geschichten passen nicht in den Rahmen des ersten Sabbats in Kapernaum.

Sie passen aber so, wie sie erzählt werden, überhaupt nicht in die geschichtliche Wirklichkeit. Das gilt vor allem von der Berufung der beiden Brüderpaare. Darin hat allerdings Holtzmann gegen Feine recht, daß niemand, der sich auf Wirklichkeit versteht, die Unanschaulichkeit dieser Erzählung für die Anschaulichkeit von Lc 5, I- II umtauschen wird. Abcr für Geschichte wird er auch diese Erzählung dann nicht nehmen. Sie ist nicht nur formell eine Neubildung der Berufungsgeschichte des Elisa, sondern auch inhaltlich in jedem Zuge allegorische Poesie. Das zeigt doch jedem, der sich auf Dichtung versteht, schon der Parallelismus der Berufung beider Brüderpaare. Und hier kann nicht etwa nur cin Glied dem andern nachgebildet sein. Hier ist alles der dichterischen Phantasie entsprossen. Oder könnte man wohl den geschichtlichen Vorgang, wie Petrus ein Jünger Jesu wurde," noch irgendwie aus dieser Erzählung „herausschälen“? Man muß sich diese Frage nur einmal ausdrücklich stellen, um die Unmöglichkeit ihrer Beantwortung zu erkennen. Auch Joh. Weiß gesteht das zu (S. 140f.), sucht aber die Geschichtlichkeit der Erzählung als ein besonders charakteristisches Moment aus der Vorgeschichte des Jüngertums der Vier dadurch festzuhalten, daß er ihm "Berührungen, Gespräche, Eindrücke" von Jesus vorhergegangen sein läßt. Aber man stelle sich nur vor, daß Jesus die Jünger, die er schon kannte, auch nur einmal so mitten aus ihrer Arbeit weg ,berufen" habe, und daß sie dann sofort alles gelassen hätten und ihm "nachgefolgt" wären! „Berufung" und "Nachfolge" sind hier deutlich aus dem christlichen Gemeindeglauben in die Geschichte der ersten Jünger Jesu übertragen. Indem Joh. Weiß zweimal zur Verdeutlichung die Bekehrungsgeschichte des Paulus anzieht, hätte er den entsprechenden geschichtlichen Moment auch für die Berufung des Petrus in seiner Bekehrungsvision sehen müssen. Auch. Petrus ist erst durch die Erscheinung des Auferstandenen zum Apostel d. h. "Menschenfischer" geworden. Wie er aber ein Jünger ( $(\mu \alpha \theta \eta \tau \eta \dot{c} c)$ Jesu geworden ist, läbt sich aus dieser Erzählung nicht erkennen. Sie ist deshalb in keiner Weise zu den „Petruserzählungen“ zu rechnen.

Der Tag von Kapernaum und der folgende Morgen bilden allerdings eine zusammenhängende Erzählungsgruppe. Aber der Zusammenhang ist, wie wir bereits sahen, künstlich, nicht geschichtlich. Die dem Evan- 
gelisten geläufigen Eingangsformeln (dreimal kaì €ứúc), sowie seine Theorie vom Messiasgeheinnis (V. 25 und 34) lassen vermuten, dab er von diesem selbst erst hergestellt ist. Er soll programmatisch die Tätigkeit Jesu als Messias darstellen, wie sie im Schlu@vers (39) bündig zusammengefaßt ist: Lehren und Dämonenaustreiben.

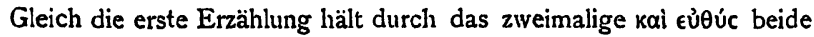
Tätigkeiten Jesu reinlich auseinander, um dann aus beiden zusammen seine Éoucía als Messias zu proklamieren. Denn Ěoucíav Éxwv heillt nicht, wie Holtzmann (H. C. ${ }^{3}$ S. II5) paraphrasiert, so allgemein „ein Lehrer vọn Gottes Gnaden", sondern es hat, wie 'auch sonst im hellenistischen Sprachgebrauch, den speziellen Sinn der göttlichen Vollmacht und bezieht sich ja auch hier auf Lehre und Wunder. Auf Jesus angewandt bedeutet es nach C. II, $28 \mathrm{ff}$. noch ganz speziell seine messianische Vollmacht. Der Beweis der Messianität Jesu lag ja für die älteste Zeit allein in seiner Auferstehung Act 2, 36, Röm 1, 3. Als man aber sein messianisches Wesen in seinem Erdenleben nachzuweisen begann, mußte man es vor allem in seiner göttlichen Vollmacht zu lehren und in seinem siegreichen Kampf gegen den Satan und dessen Dämonen tun. Ganz besonders in letzterem sah man damals die eigentliche Aufgabe des Messias nach jüdisch-hellenistischer Vorstellung. Es ist dies also auch die älteste Vorstellung von der Messianität Jesu in der Urgemeinde gewesen, die der Evangelist dann in C. $8 \mathrm{ff}$. mit einer jüngeren, durch den Einfluß des Paulus entstandenen, verbunden hat. $\mathrm{Da}$ die Vorgänge in der Synagoge diesen Messiasbeweis Jesu programmatisch darstellen sollen, geht noch aus einem doppelten hervor: Einmal wird bezüglich der Lehre Jesu nur der gewaltige Eindruck hervorgehoben, während uns kein Wort über ihren Inhalt gesagt wird. Sollte Petrus, wenn er der Berichterstatter dieser ersten Predigt Jesu gewesen wäre, davon gar nichts gesagt haben? In einem wirklich geschichtlichen Bericht hätte doch gewil davon etwas gestanden. Sodann liegt auch bei der Heilung des Besessenen der ganze Nachdruck auf dem vơn dem Dämon enthüllten Messiasgeheimnis, das durch die darauf folgende Heilung des Besessenen nur seine Bestätigung erfährt. Es soll hier nicht die Heilung eines beliebigen Kranken, sondern die Übermacht Jesu über die Dämonen und ihr Reich (vgl. auch den Plural oío $\alpha \mu \epsilon v$ ) erzählt werden. Ich kann mich für die nähere Begründung hierfür auf den Aufsatz Wredes in dieser Zeitschrift (1904 S. 169: Zur Messiaserkenntnis der Dämonen bei Markus) berufen. Gewi $\$$ hat Jesus in Kapernaum gelehrt. Wahrscheinlich hat er auch als Exorzist gewirkt 
und durch dic Kraft seiner Persönlichkeit - viclleicht auch einmal in einer Synagoge - "Besessene" gelicilt: aus der Schilderung in Mc I, 2 ff. läbt sich jedenfalls kein bestimmter geschichtlicher Kern mehr entnehmen; sie hat religiöser Poesie und nicht der Geschichte ihre Gestaltung zu verdanken.

Der solenne Abschluß der Geschichte in V. 28 zeigt, daß die Erzählung mit der folgenden nicht in ursprünglichem Zusammenhange gestanden hat. Der Evangelist hat erst beide durch das dritte kui eüuve in Verbindung gebracht und wohl auch erst die Namen der vier Jünger eingefügt, um den Zusammenhang dieser Geschichten auch mit der Berufungsgeschichte der beiden Bruiderpaare festzuhalten.

Daß der Anekdote von der Schwiegermutter des Petrus geschichtliche Erinnerung zugrunde liegt, ist an sich nicht unmöglich. Weiß (S. 146) sucht das Wunder dadurch glaubhaft zu machen, daf er die Krankheit als Nachwehen von Malaria-Wechselfieber erklärt, zu deren Überwindung Jesus der Erschöpften „durch sein kraftvoll-begeistertes Auftreten, durch die energische Ergreifung der Hand und doch wohl auch durch ermunternden Zuspruch die nötige Stärkung des Willeñ́s mitgeteilt habe". $\mathrm{Da}$ aber dem Petrus und seiner Umgebung ein solcher Zuspruch als übernatürliche Heilung habe erscheinen müssen, ist doch etwas viel behauptet. Die Erzählung setzt eine plötzliche volle Genesung der Darniederliegenden voraus, die sie zur Aufwartung der Gäste befähigte. Und eben hierin lag das Erbauliche der Anekdote: die Diakonie der Witwen ist ein bekannter Zug aus dem altchristlichen Gemeindeleben. Die Geschichte hat also jedenfalls eine Umbildung erfahren, die leichter aus der volkstümlichen Gemeindetradition als aus dem Munde des Petrus zu erklären ist. Der einzige konkrete Zug des Ergreifens der Hand beruht nicht auf persönlicher Erinnerung, sondern auf stehendem Gebrauch 5, 42. 9, 27.

Daß die nun folgende Massenheilung am Abend auf geschichtlicher Erinnerung beruhe, glaube, wer mag. Sie kehrt V. 3, 9. 3, 10. 6, $54 \mathrm{ff}$. vgl. auch $\mathrm{I}, 45.6,5.13$ wieder und bezeugt schon dadurch ihre'völlige Ungeschichtlichkeit. Zudem drängt sich in den kurzen Versen auch die sonstige Eigenart des Evangelisten förmlich zusammen: die doppelte Zeitbestimmung V. 32, die Versammlung der ganzen Stadt an der Tür V. 33, die Theorie vom Messiasgeheimnis V. 34. Weiß (S. 148) muf. das alles streichen, um dann ,áls gutbezeugte“ Tatsache übrig zu behalten, daß "unmittelbare Heilungserfolge kaum vorgekommen" sind! Solcher „Kern" ist doch wahrlich zu wurmstichig! 
Theorie des Evangelisten ist endlich auch die Flucht Jesu am frühen Morgen, die ohnehin durch den IVegfall des Wunderabends ihre scheinbare Rechtfertigung verloren hat. Auch Weiß gesteht zu, dab Jesus „im Grunde genommen durch das ganze Evangelium hindurch immer auf der Flucht vor dem Volke begriffen" ist (S. 151). Warum aber die Flucht hier gerade von höchster psychologischer Wahrheit und Überzeugungskraft sein soll, ist nicht recht einzusehen. Um an andern Orten aufzutreten, brauchte Jesus sich doch nicht heimlich bei Nacht aus dem Staube zu machen! Er wartet ja auch wieder an dem beliebten einsamen Orte, bis ihn Petrus mit seiner Umgebúng findet! Und wie konnten sie ihn schon am frühen Morgen alle suchen? Kurz, ein verständliches Bild der Szene kann man sich nicht machen, wenn man sie nimmt, wie sie ist. Sie auf Petruserzählung zurückzuführen, haben wir nicht den geringsten Anlaß.

So löst sich denn der ganze Wundertag von Kapernaum in einzelne unbestimmt geschichtliche Traditionen auf, die der Evangelist durch seine Theorie vom Messias und seinem Geheimnis zu einem Ganzen verbunden hat, ohne doch die Nähte völlig verdecken zu können. Und Weiß hat ganz recht, da $\ddot{B}$ nur unbegrenztes Vorurteil dazu bestimmen kann, in diesen Traditionen von Wundergeschichten und wunderlichen Geschichten Petruserzählungen zu vermuten.

Mehr persönliche Färbung als der Tag von Kapernaum scheint auf den ersten Blick das Petrusbekenntnis von Cäsarea Philippi zu tragen. Aber grade hier gehen schon die Meinungen unsrer Kritiker selbst über das, was zu den Petruserzählungen gehöre, weit auseinander. Während v. Soden den ganzen. Abschnitt C. 8, 27-9, I mit Leidens- und Auferstehungsweissagungen dazu rechnet, läßt Joh. Weiß nur die erste Petrusszene gelten und leitet die zweite Petrusszene ebenso wie die erste Leidensverkündigung vom. Evangelisten und nicht aus der Petrusüberlieferung ab (S. 240f.). Dagegen faßt er das Petrusbekenntnis mit der Verklärungsgeschichte als "Gruppe" zusammen, die als solche für die Geschichtlichkeit ihres Inhalts bürgen soll, während v. Soden m. E. mit Recht die Verklärungsgeschichte ganz aus der geschichtlichen Überlieferung streicht. Man sieht also gleich, wie willkürlich und subjektiv hier alles begrïndet ist.

Man wird von kritischem Standpunkte aus Joh. Weiß gewiß darin recht geben müssen, $\mathrm{da} \emptyset$ er die Leidensweissagung und damit natürlich auch die zweite Petrusszene aus der geschichtlichen Überlieferung streicht. Markus führt hier, wie Wellhausen fein gezeigt hat (Einleitung S. $79 \mathrm{ff}$.), 
einen neuen Mcssiasbegriff ein: den verklärten und himmlischen Messias des Paulus, der durch Leiden, Sterben und Auferstehen seine irdische Sendung erfullt. Diescr Messias ist, wie der Schlußvers dieses ganzen "Jüngerteils" C. 10, 45 ausdrücklich sagt, ganz nach paulinischer Auffassung nur gckommen, um zu stcrben, um sein Leben als Lösegeld für viele $z u$ lassen. Es ist klar, daß dies weder die geschichtliche, noch die ältere urchristliche Auffassung der Messianität Jesu ist. Nach der letzteren hatte Jesus, wie wir zu C. I gesehen haben, die Vollmacht zu lehren und siegreich wider die Herrschaft Satans und der Dämonen aufutreten. Beide Begriffe ließen sich nicht innerlich verbinden; der Evangelist hat sie deshalb in C. $\mathrm{I}-7$ und 8-10 nebeneinander cntfaltet; den ersteren brachte er mit, den letzteren trug ihm die urchristliche Tradition entgegen.

Wie ist nun aber bei dieser Auffassung das Petrusbekenntnis zu verstehen? Haben wir darin geschichtliche Tradition oder Theorie? Ich glaube, dal ihm in der Ortsbestimmung und auch irgendwie in der Sache geschichtliche Tradition zugrunde liegt. Aber Markus hat die Tradition so in den Dienst seiner Idee gestellt, daß sich der geschichtliche Sinn der Szene in keiner Weise mehr feststellen lassen wird. Die Fragen, ob Jesus der Messias sein wollte, ob seine Jünger ihn als solchen bekannt haben oder nicht, ob er ihr Bekenntnis angenommen oder abgelehnt hat, sind geschichtlich aus unsrer Stelle nicht mehr zu beantworten. Bei Joh. Weib (S. $226 \mathrm{ff}$.) kann man einen Eindruck davon bekommen, wie verschieden die Antwort je nach der Stellungnahme des Kritikers sein kann. Die Antwort Jesu V. 30 steht jedenfalls im Dienste der Geheimnistheorie des Evangelisten. . Dieser benutzt sie hier, um daran seine Belehrung über den leidenden Christus anzuschließen. Aber auch die Fragen Jesu erstlich nach der Meinung des Volks und zweitens nach der der Jünger sind schematisch und nicht geschichtlich. Sollte Jesus wirklich nicht gewußt haben, was die Leute und auch die Jünger von ihm denken? C. 8,27 f. ist wohl C. 6, I4f. nachgebildet. Geschichtlich festzustehen scheint nur, daß Jesus hier den Entschluß gefaßt hat, nach Jerusalem zu gehen; freilich nicht, um dort zu sterben, sondern eine Entscheidung herbeizuführen. Bei dieser Unbestimmtheit der geschichtlichen Grundlage des Petrusbekenntnisses hat man aber gewi keinen Grund, es auf direkte Petruserzählung zurückzuführen. In der Geschichte, wie wir sie haben, liegt sie jedenfalls nicht vor.

Das prophetische Auftreten Jesu als Lehrer und Exorzist, seine Wirksamkeit in Kapernaum und den umliegenden Ortschaften, die Gewinnung einiger Fischer zu vertrauten Schülern und Anhängern: das sind 
die ganz allgemeinen geschichtlichen Erinnerungen, die der galiläischen Periode im Markusevangelium zugrunde liegen. Die einzelnen Anekdoten selbst sind in der volkstümlichen Tradition entstanden und jedenfalls bis zur Aufzeichnung in unserm Evangelium so aus- und umgestaltet, daß wir bei den allermeisten auf sichere geschichtliche Erkenntnis zurzeit verzichten müssen. Irgend eine derselben auf direkte Petruserzählung zurückzuführen, ist in der Sache selbst durchaus unbegründet und ohne Bedeutung. Auch das Petrusbekenntnis ist geschichtlich nicht gesichert. Cäsarea Philippi läßt sich nur als der geographische, und zeitliche Wendepunkt der galiläischen und jerusalemischen Periode erkennen. $\mathrm{Ob}$ es auch inhaltlich in bezug auf die messianische Frage einen Wendepunkt für Jesus und seine Jünger bedeutet, ist nicht sicher $\mathrm{zu}$ entscheiden. Fest steht nur, daß es im Markusevangelium einen solchen zwischen zwei verschiedenen Messiasbildern bildet, die wir kurz als das urchristliche und paulinische bezeichnen können. Das letztere gewinnt dann von C. II an wieder die Oberhand. Ob es aber schon im Leben Jesu für ihn und seine Jünger eine Rolle spielte, ist ungewiß. Die Idee des Messiasgeheimnisses müßte dann eine andere Bedeutung haben, als ihr Wrede gibt. Sie müßte sich dann nur auf das paulinische Messiasbild beziehen, das für den Evangelisten selbst als das einzig wirkliche und geschichtliche gilt. Doch das sind Fragen, die hier nicht näher zu erörtern sind. Ich habe sie nur aufgeworfen, um zu zeigen, auf welchen Gebieten m. E. die Hauptprobleme des Markusevangeliums liegen. Es sind die der inneren Kritik, auf die Wellhausen und Wrede die Forschung gewiesen haben. Die Literarkritik hat gewiß auch noch genug Arbeit, kann aber nicht sehr weit kommen, weil sie auf dem Boden der volkstümlichen Tradition keine Nahrung mehr findet. Aber das Suchen nach Petruserzählungen sollte man gänzlich aufgeben. Es hat gegenüber dem Inhalt und der Komposition des Markusevangeliums keinen Anspruch mehr auf wissenschaftliche Bedeutung.

Es bleibt uns nur noch übrig, kurz die Frage zu erörtern, wie es zu der Sage von Petruserzählungen gekommen ist. Wir haben oben schon gesehen, dab die altkirchliche Tradition dieselben auf unser ganzes Markusevangelium bezieht, und daß sie das deshalb tut, um dem zweiten Evangelium mit der nötigen apostolischen Autorität die kirchliche Anerkennung und Geltung zu verschaffen. Abër diese Sage ist auch nicht plötzlich entstanden, sondern hatte selbst wieder ihre Anknüpfung in der volkstümlichen Tradition, die schon manche ihr besonders wichtige und wertvolle, aber vom Unglauben leicht anfechtbare Überlieferungen mit 
den Namen berühmter Apostel, insbesondere des Petrus, gedeckt hatte. Es sind dic schon oben angeführten Erzällingen von der ersten Totenerweckung, von der Verklärung Jesu, von der Zukunftsweissagung, von scinem Leidenskampfe in Gethsemane. Man wird zugeben, dab grade diese Stücke für die älteste Christengemeinde ebenso wertvoll waren, wie sic geschichtlich unglaubwürdig sind.

Für die hole Bedeutung der Verklärungsgeschichte haben wir 2. Petr. 1, $16 \mathrm{ff}$. noch im NT ein Zeugnis. Es ist also verständlich, dab man sie mit den Namen der berühmtesten Apostel zu decken suchte. Dal sie trotzdem keinen Anspruch auf geschichtliche Glaubwürdigkeit hat, bedarf hier keiner näheren Begründung. Die geschichtliche Grundlage, die ihr Joh. Wei@ zu retten sucht (S. 248), läßt grade die Hauptsache, die Verklärung Jesu und die Fimmelsstimme, fallen und ist mit ihrer unmotivierten Petrusvision des Mose und Elias noch ebenso unglaublich. Jesus hätte danach die drei Vertrauten besonders mit auf den Berg genommen, auf dem dann Petrus eine für ihn und für Jeșus völlig unerwartete Vision gehabt hätte! Der Kernpunkt der Verklärungsgeschichte muß vielmehr wegen ihrer feierlichen Vorbereitung durch Jesus von Anfang an in seiner Verklärung selbst bestanden haben, eine für die Urgemeinde gevil höchst wertvolle Bestätigung ihres Osterglaubens.

Einem ähnlichen Glaubensbedürfnisse hat auch gewiß die Jairusgeschichte ihren Ursprung zu verdanken. Der Lebensfürst mußte sich als solcher wenigstens schon einmal zu seinen Lebzeiten auf Erden erwiesen haben. Und auch dies haben wieder die drei Vertrauten mit gesehen und bezeugt C. 5, 37. Nach Joh. Weiß (S. 193) hat Jesus die Kranke allerdings nur aus einem totenähnlichen Zustande erweckt. Aber die ganze Geschichte ist nach alttestamentlichen Mustern (1. Kön 17, 17 ff. und 2. Kön 4, 8. 17 ff.) deutlich auf eine Totenerweckung angelegt: Die Bitte des Vaters V. 23, die Todesbotschaft V. 35, die Totenklage V. 38. Jesus wäre dann, wie auch seine Worte und sein Auftreten V. 36 und 39 zeigen, mit voller Überzeugung und mit absoluter Gewvißheit, eine Tote zu erwecken, in das Haus des. Jairus (= er wird erwecken!) gegangen, und nur ein günstiger Zufall hätte ihn davor bewahrt, nicht wirklich dem Gelächter der Menge anheimzufallen! Ich halte es für durchaus unerlaubt, die Geschichtlichkeit dieser Erzählung auf solche Weise retten zu wollen, die der Person Jesu wahrlich keine Ehre antut. Will man das Wunder beseitigen, un einen geschichtlichen Kern zu retten, so darf man auch die übrigen Umstände, die auf das Wunder angelegt sind, nicht bestehen lassen, sondern maß eine gänzliche Umbildung der 
Geschichte durch die Tradition annehmen. Einen genießbaren Kern wird man dann freilich nicht behalten. Wie wir die Geschichte jetzt lesen, will sie eine von Jesus beabsichtigte Totenerweckung erzählen und ihre Wahrheit durch das Zeugnis des Petrus und der beiden andern Apostel verbürgen. Wenn wir dieser Bürgschaft nicht mehr glauben, ist das eine Sache für sich; für die damalige Zeit galt sie unverbrüchlich.

Äußerst wertvoll mußte für die alte Christenheit auch eine verbürgte Zukunftsweissagung Jesu sein. Allerdings nicht die, die z. B. v. Soden aus C. I3 auf die Petrusüberlieferung zurückführt (s. o.), sondern grade die Verse $7-27$, die er daraus streicht. Nun wissen wir aber, daß diese Verse nicht von Jesus herrühren, sondern aus der jüdischen Apokalyptik; die Rede wird wohl mit Recht als ein christlich überarbeitetes Flugblatt aus der Zeit des jüdischen Krieges angesehen. Wodurch hat sie nun das Ansehen einer Weissagung aus dem Munde Jesu erlangt? Dadurch, daß sie als eine Geheimtradition an Petrus und die andern Vertrauten Jesu ausgegeben wurde V. 3 f. An diesem Beispiele ist es also noch besonders deutlich $\mathrm{zu}$ sehen, warum und wie sich die Tradition von Petrusüberlieferungen gebildet hat. Ein anonymes Flugblatt tauchte auf. Von wem stammt die Weissagung? Von Jesus. Woher weiß man das? Er hat es dem Petrus und den andern Vertrauten auf dem Ölberge gesagt, als er mit ihnen den Tempel gegenüber saß. $\mathrm{Da}$ es immer mehrere Zeugen waren, sollte wohl im Anfang das Zeugnis verstärken. Später genügte die Autorität des Petrus allein.

Die Voraussetzung für unser Verständnis der Petruserzählungen ist immer ihre geschichtliche Unglaubwürdigkeit. Aber trifft diese Voraussetzung auch für die Gethsemanegeschichte zu? Die darin ausgesprochene Stimmung der Angst und. Ungewilßheit Jesu scheint um so wahrer zu sein, je weniger sie zu der späteren Anschauung von seinem Tode als dem eigentlichen und beabsichtigten $Z$ weck seines ganzen Lebens stimmt, die auch im .Markusevangelium C. 8-10, bes. C. IO, 45 und in der Einsetzung des Abendmahls zum Ausdruck kommt. Und gewiß gibt auch diese ergreifende Szene noch in anschaulicher Darstellung die so verschiedene Stimmung Jesu und seiner Jünger "ohne alle Schminke und Salbung" wieder. Aber grade das besondere Gebet Jesu, das doch niemand gehört haben könnte, ist es hier, was die drei Vertrauten bezeugen sollen. Man darf nur nicht an das paulinische Christusbild dabei denken, zu dem der Inhalt des Gebetes nicht melr pabt; sondern man 
mul das urcluristliche Messiasbild vor Augen laben, zu dem der gewaltsame Tod Jesu im grellen Widerspruche stand, um die grofe Bedeutung des Gebctsinhaltes von Gethsemane für die Urgemeinde zu verstehen. Denn er bezeugte ihr, daß Jesus um seinen bevorstehenden Tod gewult habe, und dab er sich unter sein Geschick als ein von Gott gewolltes und zur Vollendung seines Werkes verhängtes willig gebeugt habe. Von diesem Standpunkt aus gesehen erscheint die Gestalt Jesu in Gethsemane nicht unter, sondern hoch über dem Messiasbilde der Urgemeinde. Erst für den ganz paulinischen Christus des Johannesevangeliums erschien dieses Gebet allzumenschlich und würde deshalb als seiner göttlichen Würde unangemessen übergangen.

Alle ausdrücklichen „Petruserzählungen" des Markusevangeliums entstarnmen also dem Glaubensbedürnis der Urgemeinde und sollen ihr besonders wertvolle Gedanken und Züge ihres Christusbildes mit den Namen der bekanntesten Jünger und Apostel Jesu verbürgen. An der Spitze derselben stand jedesmal das eigentliche Haupt der Urgemeinde, der Apostel Petrus. Dieser wurde schlieblich der Repräsentant und Bürge der ganzen älteren evangelischen Überlieferung, wie sie uns im Markusevangelium vorliegt. Wenn man also, wie Joh. Weiß und andere tun, der altkirchlichen Tradition von Petruserzählungen ein großes $\mathrm{Zu-}$ trauen entgegenbringt, so mübte man sich vor allem an die Stücke halten, die im Markusevangelium selbst schon als solche bezeichnet werden. Aber man darf dann auch nicht grade die Hauptsachen entfernen, um die Nebensachen zu retten: die Totenerweckung, die Verklärung Jesu, die eschatologische Weissagung, die Gebetsworte von Gethsemane. Denn gerade diese werden unter den Schutz der apostolischen Autorität gestellt. Hält man diese Hauptsachen für . Sagen, dann hat das Gerede von Petruserzählungen keinen greifbaren Sinn mehr. Es wird ja gewiß die evangelische Tradition in irgend welchem Maße auch auf Petrus zurückgehen; aber irgendwelche bestimmte Erzählungen aus seinem Munde abzuleiten, geht nicht an. Es fehlen uns nicht nur alle Mittel, sie zu konstatieren, sondern es ist auch höchst unwahrscheinlich, daß sich in unserm Markusevangelium solche noch erkennbar erhalten haben.

Damit soll aber keineswegs gesagt sein, daß der ganzen evangelischen Überlieferung die gesçhichtliche Grundlage fehle. Vielmehr hat erst Wellhausens Untersuchung wieder neu gezeigt, wie stark diese volkstümliche Tradition in palästinensischem Heimatboden wurzelt. Mit ihr verhält es sich so, wie es $\mathrm{R}$. Steck über das Christusproblem (Protest. 
Monatshefte 1903 S. $85 \mathrm{ff}$.) ausgeführt hat: „Alles Einzelne ist unsicher, das Ganze ist dennoch über jeden Zweifel erhaben." Es liegt ihm ein fester Kern zugrunde: „So wie ein Baumstamm vom wuchernden Epheu übersponnen wird, aber doch allein das ganze Gewirr von Ranken trägt, das ohne ihn $z u$ Boden fallen müßte." Ob und wie es einmal gelingen kann, den Stamm von dem Geranke zu befreien, ist freilich zur Zeit noch nicht zu sagen. Das Licht, das der Kritik in der Tradition der Petruserzählungen auf dem Wege dazu voran zu leuchten schien, hat sich leider als ein Irrlicht erwiesen. 